\title{
Towards Embedded Force Sensors in Exoskeletons for Evaluating Interaction Forces in Interfaces
}

\author{
Niclas Hoffmann ${ }^{1,2}$ 凶, Samet Ersoysal ${ }^{1}$, and Robert Weidner ${ }^{1,2}$ \\ ${ }^{1}$ University of Innsbruck, Institute of Mechatronic, Chair of Production Technology, Austria \\ ${ }^{2}$ Helmut-Schmidt-University/University of the Federal Armed Forces Hamburg, \\ Laboratory of Manufacturing Technology, Germany \\ Niclas.Hof fmann@uibk.ac.at/Niclas.Hoffmann@hsu-hh.de; \\ Samet.Ersoysal@uibk.ac.at; \\ Robert.Weidner@uibk.ac.at/Robert.Weidner@hsu-hh.de
}

\begin{abstract}
The applicability of exoskeletons in different daily and occupational settings is continually increasing. Due to varying tasks and user groups, the adaptability of exoskeletons is becoming more significant, with increasing demands for smarter devices. The implementation of force sensors in exoskeletal interfaces could be an approach for analyzing the human-machine interaction in terms of, e.g., wearing comfort, support, and motion synchronicity, as well as optimizing this interaction in real time based on the analyzed sensory data. For this, force sensors need to be embedded in interfaces, which implies the consideration of inexpensive sensors to minimize the total purchase price. However, measuring contact forces on the wearer is challenging and inexpensive flexible force sensing resistors have limited accuracy, repeatability, and stability. This paper evaluates the suitability of an interface principle working with two water capsules and two embedded piezo-resistive pressure sensors in different test scenarios derived from real exoskeletal application examples. Finally, a comparison of the capsules' inner pressures reliably detects different load conditions on the interface such as centered, edged, and shear forces. Thus, this principle seems to be suitable for further exoskeletal considerations.
\end{abstract}

Keywords: Force Sensors, Low-Budget, Exoskeletons, Human-Machine Interaction, Evaluation, Adaptability.

\section{Introduction}

Exoskeletons have become increasingly popular within the industrial sector with various applications [1]-[3] to support the user by decreasing general workloads or work in forced positions, as well as increasing working productivity due to prolonging muscle fatigue [4]. In principle, the successful application of any support system requires a merger of human, machine, and activity [5]. Thus, exoskeletons feature different properties based on their morphological structure such as path of force, structural rigidity, and actuation [3]. To assess the functionality, suitability, and impact of an exoskeleton in a certain context, an evaluation of the system should be conducted first, considering 
diverse criteria like level of support, kinematics, comfort, and safety [6]. For this, different methods ranging from biomechanical assessments to questionnaires are usually used to get a comprehensive impression of the exoskeleton's performance [7].

The implementation of force sensors in interfaces of exoskeletons may help to conduct a multicriterial evaluation of exoskeletons using only one tool. Statements concerning level of support, control and dynamics, situation and intention detection, path of force as well as the design, fit and comfort of its interfaces are therefore possible.

If such sensors are incorporated in the interface, the sensory data may be analyzed in real-time and initiate corresponding adjustments of the exoskeleton. This may improve human-machine interaction and acceptance of exoskeletons equipped with force sensors. Additionally, this approach includes the use of inexpensive sensors in order to keep total cost of the exoskeleton at a competitive level. Overall, it depicts one way for exoskeletons to become smarter devices by improving their automatic adaptability to different working environments, tasks, and users.

In principle, the accurate measurement of contact forces on human interfaces is challenging [8] and requires diverse sensor characteristics like linearity, low creep, low temperature sensitivity, flexibility, and repeatability [9]. When focusing on inexpensive sensors for this application, only piezo-resistive force sensors seem to be suitable [10]. Diverse approaches have already been made using different Force Sensing Resistors (FSRs) [8], [11], however these sensors usually suffer from low linearity, stability, repeatability, high drifts and hysteresis [10], [12]-[14]. In this context, commercially available pressure sensor pads are also unsuitable due to their high purchase costs and their complexity for further processing sensor data in real-time.

The use of high-resolution pressure sensors instead of FSR seems to be beneficial for measuring surface pressure on interfaces [15], whereby the average pressure on the interface divided by the interface region implies the total support force of the exoskeleton [14]. In this paper, different test scenarios derived from real exoskeleton applications evaluate the general suitability of a developed exoskeleton interface principle with two water capsules and two embedded pressure sensors. These scenarios mentioned in chap. 3.2 may simulate possible load conditions on exoskeleton interfaces to assess the general human-machine-interaction and reveal recommendations for further actions.

\section{Interface Prototype with Embedded Pressure Sensors}

The developed interface prototype measures pressure conditions within a flexible capsule with the help of embedded pressure sensors. If a load occurs on the interface, the flexible capsule is compressed and its inner pressure rises, which is detected by the pressure sensor. Overall, the function of the flexible capsule can be broken into twopart: a) pressure detection and b) replacement of foam for bolstering the interface by working as a cushion itself.

In its basic structure, the prototype is inspired by the work of Lee et al. [15], who designed a prototype for a pressure sensor system based on a "micro-sensor inside a flexible capsule filled with a pressure transmissible fluid". Their prototype was built 
out of a plastisol bladder as a flexible capsule, laminated on an aluminium plate, using hair gel as transmissible fluid, and the pressure sensor MPX2300D.

Here, the interface prototype uses the above basic construction principle, but a cast silicone capsule, water as transmission fluid, the modern high-resolution pressure sensor MS5803-01BA, and a second aluminium plate for detachable clamping of the flexible capsule onto the aluminium baseplate. In addition, the prototype is expandable to additional flexible capsules with multiple pressure sensors. This may be useful, as apart from magnitude and duration, the pressure distribution is of special interest for human interfaces, which is caused by changes in human soft tissue or mismatches of exoskeletal and human axes [11], [14], [16]. In addition, the force magnitude may be solely assessable via power curves of the exoskeleton actuation or simulated human models. In this approach, the prototype does not feature a third chamber in the middle of the interface, due to load usually shifting towards its edges [11]. Furthermore, this approach is supported by an economic perspective limiting the total amount of sensors.

\subsection{Construction}

The construction of the interface prototype is illustrated in the figure below. The left side shows its lateral cross section at sensor level, the right side its total view presented in an exploded view drawing.

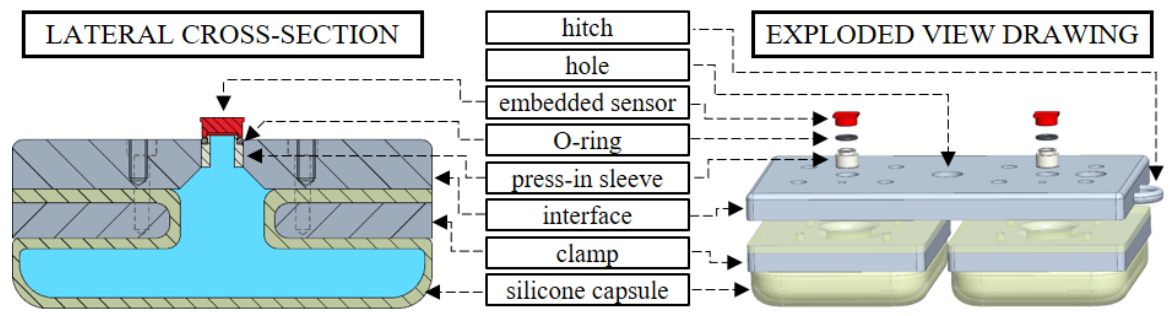

Fig. 1. Construction of the interface prototype with embedded pressure sensors.

The constructive base of the interface prototype is a milled rectangular aluminium plate with the dimensions of $100 \times 60 \times 10 \mathrm{~mm}$, that represent the exoskeletal interface. For later load experiments, holes in the center and the right edge are drilled into the plate, and a hitch on the right lateral face is installed. On the interface, the two pressure sensors are placed in two transfixions on a pressed sleeve and clamped with a crossbar (not illustrated in Fig. 1). This connection is sealed with an O-ring on the outer diameter of the metal cap of each sensor [17].

The used pressure sensor MS5803-01BA, which is based on leading MEMS technology and placed on a breakout board for an easier junction, is a piezo resistive, highresolution, low power, and economical altimeter sensor with adjustable resolution. For the used resolution of $0.012 \mathrm{mbar}$, the response time is 8.22 milliseconds. The accuracy in the used range is \pm 1.5 mbar. Due to a gel protection, the sensor can measure pressures in different mediums like liquids or air. For further technical details, see [17]. In this set-up, the sensor runs on an Arduino UNO via $\mathrm{I}^{2} \mathrm{C}$ communication. 
The flexible capsule needs to be shapeable, resilient, sealed, and skin-friendly, which is achieved by the multi-purpose silicone "Dragon Skin 20" [18]. For molding the capsule, this two-component silicone is mixed, stirred, evacuated to release air bubbles, and cast in a milled mold. The benefits of the molded capsule are a return to its original form without load, no recorded irritabilities in case of longer skin contact, and an easy adaptability of its shape to different dimensions of exoskeletal interfaces. The dimensions of each capsule are $60 \times 47 \times 10 \mathrm{~mm}$ and the wall thickness is $1.0 \mathrm{~mm}$ on the sides and $1.5 \mathrm{~mm}$ on the bottom and the top. After curing, each capsule is positioned under the corresponding transfixion of the interface and clamped by a second milled aluminium plate with four mounting screws, respectively. After that, the capsule is filled with water, vented, and sealed with the pressure sensor by fixing the screws of the crossbar.

\subsection{Calibration of the Pressure Sensors}

First, the prototype is placed on a hard surface, e.g., a wooden table. The calibration weights are centered on a $3 \mathrm{D}$ printed sheet that is stuck into the center hole on the aluminium plate, in order to consistently distribute the load on each silicon capsule. The following calibration process is derived from [8]. Under barometric pressure and room temperature with relative humidity, the interface is loaded and unloaded in the center with three weights $(200 \mathrm{~g}, 350 \mathrm{~g}, 500 \mathrm{~g})$ three times with a duration of five seconds each and a sampling rate of 50 milliseconds. Three pressure values after foursecond measurements are taken and averaged. Based on the respective pressure gradient of each load against the pressure without load, a linear three-point calibration is made. The quality of the linear regression using this method has proven very sufficient $\left(\mathrm{R}_{\text {sensor1 }}=0,9997\right.$ and $\left.\mathrm{R}_{\text {sensor2 }}=0,9999\right)$ and even a little improved compared to [15]. This suggests that the linear performance of each sensor is very good in this measurement range. For later interpretation, the load with weights in gram $(\mathrm{g})$ is translated to an acting vertical force in Newton $(\mathrm{N})$.

\section{Experiments}

Since Lee et al. [15] already demonstrated the benefits of this measurement principle against other commercially available, low-cost force sensors in terms of linearity, temperature effect, hysteresis, reliability and creep, the key aims of this work are to place these within the context of exoskeletons:

- Is the developed prototype suitable for assessing loads, load distributions, and shear forces on an exoskeletal interface?

- How many pressure capsules are necessary and in what arrangement, to detect different load conditions reliably? From an economic perspective, the aim is to keep the number of sensors to a minimum.

For this, different experiments are derived from possible application scenarios for an exoskeleton for the upper extremities. Each experimental set-up uses varying weights and weight positions to represent different support forces of an exoskeleton, or load conditions caused by different movements and tasks. 


\subsection{General Test Set-Up}

In order to focus on the principal applicability, the prototype is tested in a controlled laboratory setting. In reality, the supportive force of an exoskeleton affects the human soft tissue through the interfaces, where the interface distributes the force as surface pressure to the contact area on the body. Various load conditions, distributions, and shear forces may occur (see Fig. 2). In the lab experiments, this is simulated with different weights placed on a $3 \mathrm{D}$ printed plate and positioned in the center and on the edge of the interface. A spring scale simulates shear forces. The human soft tissue is modeled with two silicone layers with different shore hardness [19] (upper layer: "Ecoflex 0010", lower layer: "Dragon Skin 20").

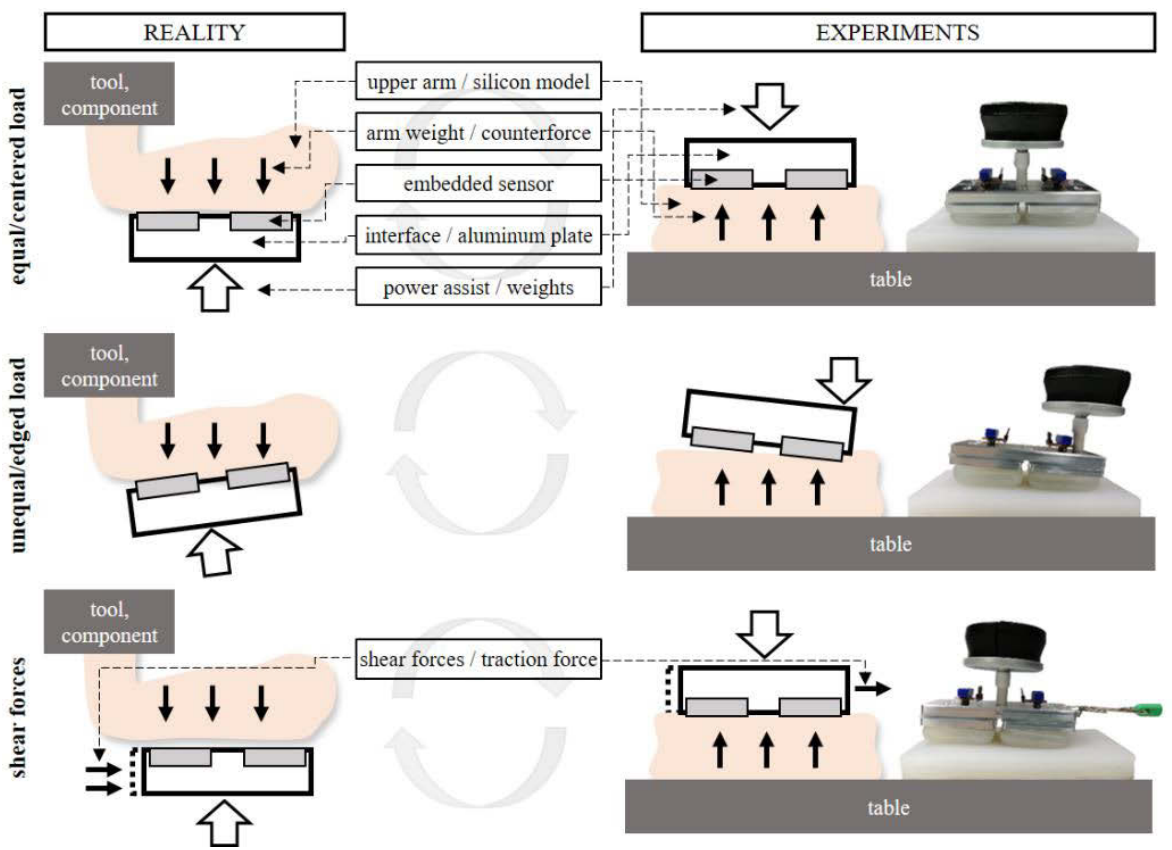

Fig.2: Derivation of the general test set-up and scenarios from real exoskeletal applications on the example of the upper arm.

\subsection{Test Scenarios}

Test scenario A "Equal Continuous Constant Load" describes a task that is performed for a prolonged period of time in the same physically demanding posture (i.e. the complex electronic cable insertion in car doors, which is performed overhead/above shoulder level). This is tested with a constant load of $4.9 \mathrm{~N}$ placed only in the center of the interface for 120 seconds. Lee et al. [15] tested such a scenario for 144 seconds.

Test scenario B "Continuous Decreasing Load" considers a continuous load on the center resp. edge for a prolonged period, which gradually decreases over time. One example may be the emptying of goods from a large box/container and placing them 
separately onto shelves or into different storage spaces, so that the weight of the load carried decreases. Another example is the installation of pipes on a ceiling with clamps, so that the weight of the pipe decreases with every fixed clamp. In the tests, the interface is loaded with $5.9 \mathrm{~N}$ to begin with, and then slowly decreases to $5.8 \mathrm{~N}, 5.4 \mathrm{~N}, 4.9 \mathrm{~N}$, and $3.9 \mathrm{~N}$ after six seconds each. The respective gradient deliberately varies.

Test scenario C "Repetitive Constant Load" represents a repetitive task at head level or above, e.g., driving screws in a ceiling, with the same weights of tools or other components. If users raises their arms, the exoskeleton may support them, hereby applying surface pressure to the human tissue. If users lowers their arms, no pressure is applied due to the lack of load on the upper extremities. In the simulation, the aluminium plate is repetitively loaded five times on the center resp. edge for three seconds with $3.9 \mathrm{~N}$. A pause of three seconds is conducted in between.

In the test scenario D "Repetitive Varying Load", the ability of the prototype of measuring varying repetitive loads is evaluated. In practice, these loading conditions may appear, if the user of an exoskeleton needs to randomly handle light and/or heavy packages (i.e. working in logistics). The support of especially active exoskeletons is usually adaptable, which may lead to varying loads. In the test set-up, the interface is consecutively loaded on the center resp. edge with $4.9 \mathrm{~N}, 0.2 \mathrm{~N}, 3.9 \mathrm{~N}, 1.5 \mathrm{~N}$, and $4.9 \mathrm{~N}$ for a duration of three seconds. A pause of three seconds is conducted in between.

Test scenario E "Repetitive Varying Shear Forces" evaluates the ability of the prototype to assess shear forces during motion, that may be a cause for friction and may even lead to skin abrasions. Shear forces may be an indicator for a poor fit and consequently lead to rejection of the exoskeleton. In the test, the interface is vertically continuously loaded with $4.9 \mathrm{~N}$ and in addition repetitively loaded horizontally in longitudinal resp. lateral direction of the interface, with a varying traction force of $2.0 \mathrm{~N}, 1.0 \mathrm{~N}$, $2.9 \mathrm{~N}, 4.9 \mathrm{~N}$, and $2.9 \mathrm{~N}$. A shaped metal crossbar prevents any rolling of the interface to focus only on the influence of horizontal motions of the interface on each capsule pressure.

\section{$4 \quad$ Results}

For every test scenario, the different sensory data are stored and plotted in Matlab R2018b and presented in Fig. 3. Based on the inner pressure and the calibration of each sensor, the respective load conditions may be concluded.

Both sensors accurately measure near the same pressure, if the interface is equally loaded with the same weight for a prolonged period of time (see scenario A). Both sensors also measure similar pressure for equal repetitive loads (see test-retest scenarios C and D). In case of an equal load, both sensory values add up to near the applied load. If an unequal load occurs, the inner pressure of the capsule underneath the load measures the applied load and additionally rises with almost the same gradient like the equivalent of decrease in pressure occurring to the other capsule (see scenario B, C, D). Test scenario $\mathrm{B}$ shows that the sensors may even detect marginal changes in load as small as $0.1 \mathrm{~N}$, and further, measure varying consecutive loads with high accuracy (see scenario D). 

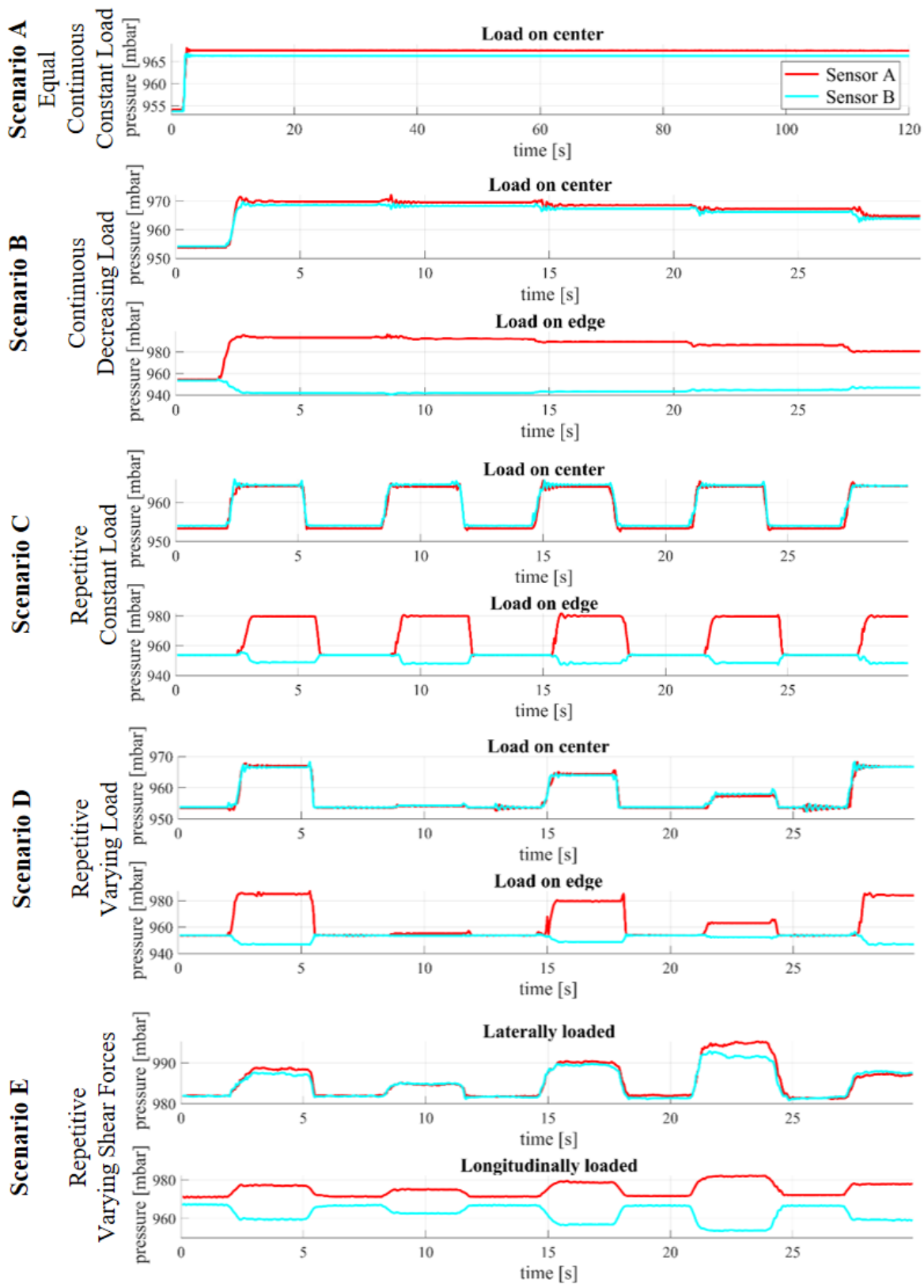

Fig. 3: Results of the different test scenarios. The abscissa shows the passage of time and the ordinate the inner pressure in the capsules measured by the pressure sensors. 
However, small loads may be detected as a rise in the inner pressure but may also be wrongly interpreted as a negative or decreasing force (see scenario D). If horizontal shear forces longitudinally act on the interface, the value of the anterior sensor to the location of applied load rises slightly, while the value of the posterior sensor decreases at a larger rate - this may be true even for small forces. This effect does not appear for laterally acting shear forces (see scenario E) and all vertical loads (see scenario B-D) on the interface.

\section{Discussion}

An analysis of the results shows that the interface prototype has a good stability, repeatability, and accuracy in its measurements. Even marginal load changes may be detected, however, their interpretation may be prone to error, due to the constant of the linear regression, on which the calibration of the sensors is based. Thus, although a small load is usually detected, it may be wrongly interpreted as a small decrease in load. However, a comparison of both sensory data may help to identify and objectively interpret the following load conditions on the interface, which may include recommendations for further actions.

For the following ratios, the respective interpreted load identification is presented. The " $X$ " represents a by an unknown factor changing variable, dependent on the corresponding load condition:

- pressure $1 \approx($ applied load $) / 2$ and pressure $2 \approx($ applied load $) / 2$ :

The load is near equally distributed. No need of action, the interface fits well.

- pressure $1 \approx$ applied load $+X$ and pressure $2 \approx-X$ :

The load is unequally distributed, leading to load peaks on the surface area covered by the capsule with a positive value (here pressure sensor 1). The exoskeleton does not fit well and there may be a possibility for perceived discomfort. Adjustments are recommended.

- pressure $1 \approx-$ applied load $+X$ and pressure $2 \approx X$ :

Horizontal shear forces act longitudinally upon the interface, which may lead to a displacement of the interface. The exoskeleton does not fit well and the possibility of abrasions exist. Adjustments are recommended.

With two capsules, the prototype is only capable of detecting load distributions and shear forces on the longitudinal axis of the interface. For statements on the lateral axis, two additional flexible capsules must be implemented on the interface in this direction. This would enable the detection of any spatial load on the interface. At this point, the benefit of an additional centered capsule requires further investigation.

However, the interface prototype depends on the barometric pressure, as the gradient against the barometric pressure is translated into respective load. Thus, the prevailing barometric pressure needs to be detected prior to each measurement to ensure accurate results. In addition, the gravity of water in the capsule has an influence on the interpreted load as well. This may occur, if the interface spatially rotates while performing certain tasks and the sensor does not remain in its vertical orientation, as it was in the 
calibration. Here, the implementation of an Initial-Measurement-Unit (IMU) on the interface may help calculate an offset. Alternatively, air may be used to replace the water medium, however this could lead to other limitations and challenges, particularly in sealing the entire interface airtight.

Finally, it must be acknowledged that the test set-up itself presents some limitations. First, the model of the human tissue used in the above study is fairly consistent, which is not always the case when working with human subjects due to factors such as varying tissue and bone structures. The loads used may also not be representative for all possible exoskeletal scenarios. However, as long as a mechanical load test of the interface prototype is pending, the loads are limited to $5.9 \mathrm{~N}$ in total, for safety reasons. The planar shape of the interface prototype and the tissue model are also abstract, acknowledging curvatures and uneven surface structures in reality for both. Finally, the environmental influences on interfaces vary in terms of temperature and humidity, which are not considered in the controlled laboratory setting.

\section{Conclusion}

In this paper, the suitability of an interface prototype with embedded pressure sensors for assessing and optimizing the human machine interaction in the context of exoskeletons is evaluated. In five test scenarios, it has been shown that this prototype is capable of reliably detecting different load conditions, between an interface and a model of human tissue.

In the future, two main areas should be focused on: the construction and the analytical ability of the interface prototype. First, a load and repeatability test of the entire prototype should be conducted, especially of the silicon capsule. Efforts should also be made to install and test this principle on curvatures, as most exoskeletal interfaces are not planar. On the other hand, the prototype should be tested on humans under real conditions and embedded in exoskeletal interfaces, whereby the influence of wearers' body temperature and sweat as well as the ability of replacing foam bolstering with the flexible capsule may be a matter of interest. The favored level of support for diverse tasks or critical pressure levels in terms of subjective perceived comfort may also be considered. At this point, the evaluation may be extended to health issues by looking at blood flow [20] or oxygen supply beneath the pressured surface [21] in association with near infrared spectroscopy (NIRS).

\section{References}

1. Fox S, Aranko O (2019) Exoskeletons: Comprehensive, comparative and critical manufacturing performance. J Manuf Technol Manag.

2. de Looze MP, Bosch T, Krause F, Stadler KS, O'Sullivan LW (2016) Exoskeletons for industrial application and their potential effects on physical work load. Ergonomics 59: 1-11.

3. Otten B, Weidner R, Argubi-Wollesen A (2018) Evaluation of a Novel Active Exoskeleton for Tasks at or Above Head Level. IEEE Robot Autom Lett 3: 2408-2415.

4. Weidner R, Karafillidis A (2018) Distinguishing Support Technologies. A General Scheme 
and its Application to Exoskeletons. In: Developing Support Technologies - Integrating Multiple Perspectives to Create Assistance that People Really Want. Springer Verlag.

5. Weidner R, Kong N, Wulfsberg JP (2013) Human Hybrid Robot: A new concept for supporting manual assembly tasks. Prod Eng 7: 675-684.

6. Hoffmann N, Argubi-Wollesen A, Linnenberg C, Weidner R (2019) Towards a Framework for Evaluating Exoskeletons. In: Production at the leading edge of technology: 441-450, Springer Verlag.

7. Argubi-Wollesen A, Weidner R (2018) Adapting to Users' Physiological Preconditions and Demands by the Use of Biomechanical Analysis. In: Developing Support Technologies - Integrating Multiple Perspectives to Create Assistance that People Really Want, Springer Verlag.

8. Wettenschwiler PD, Stämpfli R, Lorenzetti S, Ferguson SJ, Rossi RM, Annaheim S (2015) How reliable are pressure measurements with Tekscan sensors on the body surface of human subjects wearing load carriage systems? Int J Ind Ergon 49: 60-67.

9. Ferguson-Pell M, Cardi M (1992) Pressure mapping systems. TeamRehab Rep: 26-32.

10. Giovanelli D, Farella E (2016) Force Sensing Resistor and Evaluation of Technology for Wearable Body Pressure Sensing. J Sensors.

11. Levesque L, Pardoel S, Lovrenovic Z, Doumit M (2017) Experimental comfort assessment of an active exoskeleton interface. In: IEEE 5th International Symposium on Robotics and Intelligent Sensors: 38-43.

12. Lebossé C, Renaud P, Bayle B, de Mathelin M (2011) Modeling and Evaluation of LowCost Force Sensors. IEEE Trans Robot 27: 815-822.

13. Hollinger A, Wanderley MM (2006) Evaluation of Commercial Force-Sensing Resistors. Int Conf New Interfaces Music Expressions.

14. Almassri AM, Wan Hasan W, Ahmad S, Ishak AJ, Ghazali AM, Talib DN, Wada C (2015) Pressure sensor: State of the art, design, and application for robotic hand. J Sensors.

15. Lee NKS, Goonetilleke RS, Cheung YS, So GMY (2001) A flexible encapsulated MEMS pressure sensor system for biomechanical applications. Microsyst Technol 7: 55-62.

16. Sposito M, Toxiri S, Caldwell DG, Ortiz J, De Momi E (2018) Towards Design Guidelines for Physical Interfaces on Industrial Exoskeletons : Overview on Evaluation Metrics. Int Symp Wearable Robot.

17. TE Connectivity (2017) MS5803-01BA.

18. Smooth-On (2018) Dragon Skin Series Technical Bulletin.

19. Trotta TN, Island M, Trotta JA, Lowe S (2016) ( 12 ) United States Patent.

20. Holloway GA, Daly CH, Kennedy D, Chimoskey J (1976) Effects of external pressure loading on human skin blood flow measured by 133Xe clearance. J Appl Physiol 40: 597-600.

21. Sangeorzan BJ, Harrington RM, Wyss CR, Czerniecki JM, Matsen FA (1989) Circulatory and mechanical response of skin to loading. J Orthop Res 7: 425-431. 
Open Access This chapter is licensed under the terms of the Creative Commons Attribution 4.0 International License (http://creativecommons.org/licenses/by/4.0/), which permits use, sharing, adaptation, distribution and reproduction in any medium or format, as long as you give appropriate credit to the original author(s) and the source, provide a link to the Creative Commons license and indicate if changes were made.

The images or other third party material in this chapter are included in the chapter's Creative Commons license, unless indicated otherwise in a credit line to the material. If material is not included in the chapter's Creative Commons license and your intended use is not permitted by statutory regulation or exceeds the permitted use, you will need to obtain permission directly from the copyright holder. 